\title{
Prescriptive Concepts for Advanced Nuclear Materials Control and Accountability Systems
}

\author{
W. J. Whitty \\ R. B. Strittmatter \\ W. Ford \\ R. M. Tisinger \\ T. H. Meyer*
}

\section{DISCLAIMER}

\begin{abstract}
This report was prepared as an account of work sponsored by an agency of the United Staits Government. Neither the United States Government nor any agency thereof, nor any of efieir employees, makes any warranty, express or implied, or assumes any legal liability or responsibility for the accuracy, completeness, or usefulness of any information, apparatus, product, or process disclosed, or represents that its use would not infringe privately owned rights. Reference herein to any specific commercial product, process, or service by trade name, trademark, manufacturer, or otherwise does not necessarily constitute or imply its endorsement, recommendation, or favoring by the United States Government or any agency thereof. The views and opinions of authors expressed herein do not necessarily state or reflect those of the United States Government or any agency thereof.
\end{abstract}

"Electronic Data Systems Corporation, 2155 Louisiana NE, Suite 9100, Albuquerque, NM 87110-5414. 
$\begin{array}{lll}\text { ABSTRACT } & 1\end{array}$

I. INTRODUCTION 2

II. CONCEPTUAL STRUCTURE OF A DISTRIBUTED NUCLEAR MC\&A SYSTEII

A. Distributed Processing in Nuclear Materials Accountability

B. Centralized vs Distributed Data Processing

C. Distributed Systems Architecture

1. Interprocessor Communication

2. Interprocessor Cooperation

3. Topology of Distributed Systems

D. Systems Design

E. A Preliminary Configuration for a Distributed Nuclear MC\&A System

Design Considerations for the Preliminary Conf iguration

1. I/O Device Use

2. Disk Storage

3. Computer Security

4. Data-Base Protection

5. Routine Reporting

6. Special Reporting

7. System Performance

8. Special-Purpose Software vs Commercial DBMSs

III. SYSTEM DEVELOPMENT

A. Hardware Selection 16

B. Networks

C. Data-Processing Software

$\begin{array}{ll}\text { IV. INTEGRATED SAFEGUARDS } & 18\end{array}$

V. RECOMMENDATIONS FOR SAFEGUARDS R\&D 18

VI. CONCLUSIONS 20

$\begin{array}{lr}\text { ACKNOWLEDGMENTS } & 20\end{array}$

$\begin{array}{lr}\text { REFERENCES } & 21\end{array}$ 


\section{EXECUTIVB SUIMARY}

Recent advances in data-processing and communications technology coupled with rapidly declining costs have made possible a new era in the design of advanced nuclear materials control and accountability (MC\&A) systems. Networking- and distributed-processing hardware and sof tware have the potential of greatly enhancing transaction-based MC\&A systems, both from safeguards and process-operations perspectives, while allowing timely integrated safeguards activities and enhanced computer security at reasonable cost. The purpose of this report is to review distributed-processing technology applied to transaction-based MC\&A systems and to identify areas requiring safeguards research and development.

The informational requirement related to the need for managing, controlling, and protecting nuclear materials is to keep timely information on the location and status of these materials and on personnel access to these materials. This information, generated from physical security and from materials control and accounting activities, forms the basis for detection of unauthorized actions affecting the facility and, alternatively, provides positive assurance that no theft or diversion has occurred. Process data are useful for production scheduling and reporting and longterm planning. In a weli-designed integrated safeguards system, much of the safeguards data may be obtained from process data. Even in a wellintegrated safeguards system, there is a functional division of informational requirements between safeguards and processing. The safeguards information will pertain to the entire site. However, each different processing location will have unique requirements. Other process information, relating to the overall processing complex, will be required for production planning and forecasting, preparation of reports, etc. Consideration must be given to long-term information requirements as we 11 as current ones to propose an architecture for a distributed system.

The logical structure we selected for a preliminary configuration was based on the above considerations and on a hierarchical reporting structure for an integrated safeguards program. The hierarchical reporting structure derives from the U.S. Department of Energy (DOE) requirements for central responsibility for a materials management program encompassing all organizations either handling or responsible for nuclear materials. tasks:

The minimal MC\&A system of interest should perform the following

(1) data collection from instruments;

(2) validation of transaction data on entry;

(3) access contro1, data reduction, and storage;

(4) local MC\&A functions such as tracking material; and

(5) global MC\&A functions such as reporting to DOE.

In our distributed system the above tasks are performed in computers at various levels: low-leve1 machines participate in tasks 1,2 , and 3; intermediate-level machines participate in tasks 3 and 4; and the highlevel machine participates in tasks 3, 4, and 5. The logical structure of the system depends heavily on MC\&A requirements, the characteristics of the data, security considerations, and the information flow among application components. If near-real-time response has a high priority, the user should be able to do near-real-time MC\&A functions without degrading system performance. This has an impact on the data-acquisition systems, the data-base system, the network, and the user's software interface. 
Security is a special concern where networks and user-friendly modes of operation are used. A distributed system provides increased potential accessibility to the data and requires more care to protect the data from unauthorized disclosure, modification, and destruction. The insider threat poses a particular concern to the security of the safeguards information systems. The widespread use of intelligent terminals and microprocessors can allow access to the data base by applications programs, query languages, report generators, and data-base management system (DBMS) utilities. Relational DBMSs pose some security problems because they employ nonprocedural languages, which allow a user to access data without knowing the complex logical or physical structure among the data items. One of the most common weaknesses of many DBMSs to penetration is that they run under control of operating systems. We will fully investigate the security measures that need to be employed.

The culmination of this work should be a set of design criteria for implementing a distributed MC\&A system either alone or as a part of an integrated safeguards system. The application of the concepts described here would require tailoring to meet site-specific functional requirements. 
PRESCRIPTIVE CONCEPTS FOR ADVANCED NUCLEAR MATERIALS CONTROL AND ACCOUNTABILITY SYSTEMS

\author{
by \\ W. J. Whitty, R. B. Strittmatter, W. Ford, R. M. Tisinger, \\ and T. H. Meyer
}

\begin{abstract}
Networking- and distributed-processing hardware and software have the potential of greatly enhancing nuclear materials control and accountability (MC\&A) systems, from both safeguards and process operations perspectives, while allowing timely integrated safeguards activities and enhanced computer security at reasonable cost. A hierarchical distributed system is proposed consisting of groups of terminals and instruments in plant production and support areas connected to microprocessors that are connected to either larger microprocessors or minicomputers. These micros and/or minis are connected to a main machine, which might be either a mainframe or a super minicomputer. Data acquisition, preliminary input data validation, and transaction processing occur at the lowest level. Transaction buffering, resource sharing, and selected data processing occur at the intermediate level. The host computer maintains overall control of the data base and provides routine safeguards and security reporting and special safeguards analyses. The research described outlines the distribution of MC\&A system requirements in the hierarchical system and distributed processing applied to MC\&A. Implications of integrated safeguards and computer security concepts for the distributed system design are discussed.
\end{abstract}




\section{I . INTRODUCTION}

Nuclear materials safeguards at Department of Energy (DOE) sites are a complex task including physical security, materials control, and materials accounting. Recently, the need to integrate these activities to meet DOE safeguards objectives has been recognized. Also, information not traditionally associated with safeguards activities, including process control and health and safety information, is beginning to be utilized as part of an integrated safeguards approach. To implement the emerging integrated safeguards systems will require developing effective, costeffective information systems. The purpose of the report is to review distributed-processing technology applied to transaction-based materials control and accounting (MC\&A) systems and to identify areas specific to the safeguards application requiring research and development. We placed the emphasis of this report on the MC\&A subcomponent of nuclear material safeguards, while recognizing that the subcomponents must be integrated to produce a good design.

MC\&A technology consists of nondestructive assay (NDA) instruments, materials access-control instrumentation, in-line and on-line process instrumentation, communication systems, data-processing hardware, and software for handling transactions, routine reporting, ad hoc queries and analyses, and statistical analyses. The availability and development of new data-processing and communications technology have made possible a new era in the design of advanced MC\&A systems. The just-emerging distributedprocessing hardware and software have the potential of greatly enhancing transaction-based MC\&A systems, both from safeguards and security perspectives and from process control and operations considerations.

Transaction-based systems relate to operational day-to-day control activities such as shipments, receipts, shipping documents, inventory reporting and control, production control, routine reporting, and special reporting. The trend in the data-communications and-processing industry, both in hardware and in software, is to provide distributed capability for transaction-based systems. The purpose of this research is to determine whether distributed-processing technology can further the capability of MC\&A systems, and, if so, how a prototype system would be constructed and used.

A distributed-processing system is a collection of logically and physically interconnected processing elements, either located together or geographically separated, that cooperatively share the computing functions of the organization. A distributed system is not merely a network of computers dedicated to separate but mostly unrelated functions. For a group of computers to constitute a distributed system, they must work together to some degree. Finally, a distributed system is not merely a set of geographically separated terminals accessing data on a central machine because the terminals cannot function autonomously.

The remainder of this report discusses

(1) distributed processing in nuclear MC\&A,

(2) centralized vs distributed data processing,

(3) distributed systems architecture,

(4) systems design,

(5) a preliminary configuration for a distributed nuclear MC\&A system and design requirement considerations, 
(6) systems development,

(7) integrated safeguards,

(8) recommendations for safeguards research and development (R\&D), and

(9) conclusions.

\section{CONCEPTUAL STRUCTURE OF A DISTR IBUTED NUCLEAR MCEA SYSTEM}

Recent advances in computer networking hardware and software have made decentralized files and decentralized data processing with the sharing of files a reality. Common use of distributed processing with heterogeneous hardware and software requires software products that allow easy communication among machines, with much of this communication transparent to the end-user. Some of these products are starting to appear on the market. The focus of this report is on structuring a distributed MC\&A system.

\section{A. Distributed Processing in Nuclear Materials Accountability}

The primary general safeguards objectives are to protect the general public and facility employees from dangers that could arise from misuse of the facility's equipment and/or its nuclear material and to monitor the personnel access to these materials. The informational requirement related to this objective is to keep timely information on the location and status of nuclear materials and on the personnel access to these materials. This information, either alone or with additional information, can aid in the detection of unauthorized actions affecting the facility and alternatively provide positive assurance that no theft or diversion has occurred. The data collected are of little use for accountability unless the safeguards personnel can convert the data to information and analyze this information accurately, easily, and rapidly.

Distributed integrated safeguards systems have been envisioned consisting of groups of NDA instruments and terminals in plant production and support areas connected to minicomputers that in turn were connected to a main computer. The functions of the main computer included (1) maintenance of materials and personnel inventory data bases, (2) maintenance of a materials control program data base, (3) processing of inquiries and report generation, and (4) provision and analyses of near-real-time materials accounting data. Minicomputers, serving as preprocessors, acted as instrument-control devices and processed data from terminals and measurement instruments. These processed data would be used to update the comprehensive data base on the main computer. This concept freed the main computer from dealing with either instruments or transactions directly, thus releasing the main computer for other activities. 1

Microcomputers are now powerful and relatively inexpensive. There a1so have been rapid advances and price reductions in storage device technology, video display terminals, and software for microcomputers and minicomputers. Rapidly following the widespread availability of microcomputers packaged as personal computers (PCs) came more powerful microprocessorbased machines generally known as technical workstations. These workstations have several times the processing speed of most PCs and many times their usual memories. Depending on the application, technical workstations, as well as PCs and other microcomputers, could serve the same purpose as the minicomputers mentioned above. 


\section{B. Centralized vs Distributed Data Processing}

Centralized data processing uses one or several computers at one location interfaced with a number of peripherals and input and output (I/0) devices that may be separated geographically. Historically, centralized systems allowed the use of advanced file manipulation and data-management systems. The data were stored and processed in one location where economies of scale and security were important considerations. However, the production of even the simplest reports required professional programmers.

The reduction of computer system costs, advanced sof tware availability for smaller computers, advances in data-communications technology, increased reliability, increased system responsiveness, the potential for incremental growth, and the potential of resource sharing contributed to the operational feasibility of distributed processing. How would these factors apply to nuclear materials accountability?

An MC\&A system should, at least, perform the following tasks:

(1) data collection from instruments;

(2) validation of transaction data on entry;

(3) access control, data reduction, and storage;

(4) loca1 MC\&A functions such as tracking material; and

(5) global MC\&A functions such as reporting to DOE.

Strictly speaking, a centralized MC\&A system could be a single, large machine performing all of the above tasks simultaneously. A centralized system is designed to handle a limited number of $I / 0$ devices, thereby restricting the amount of information it could receive. Increasing the number of $I / O$ devices could place an additional load on the central processing unit (CPU) resulting in longer response time.

The advantages of a centralized system include the following:

(1) The elimination of the complications caused by the network. Distributed file systems, distributed data-base management systems (DBMSs), distributed resource allocators, etc., are still in the early development state.

(2) The elimination of the compatibility issue. Because there is only one machine, there are never problems with incompatible data types, protocols, etc.

If the plant is small enough and the near-real-time measurements are not too demanding, a centralized system could do the job and eliminate the networking complexity.

A distributed MC \&A system would perform the above functions by having local machines assigned to do an appropriate part of the total operation. The advantages of a distributed system include the following.

- A distributed system is more expandable than a centralized one. Because the former is a collection of autonomous machines, a good design will allow for the addition of add-ons. By enlarging existing networks and forming networks of networks, the size of the system can be expanded enormously. Conversely, a centralized system is rigidly limited to the number of I/O ports it can support and the number of concurrent processes it can run without unacceptable performance degradation. Upgrading to a larger machine may be very painful because of software conversion tasks, and it does not provide an ultimate solution. Further growth can cause the expansion probiem to recur. 
- A distributed system may employ special-purpose hardware to meet specific needs. The addition of such devices amounts to developing the proper drivers/interface units to the local area network (LAN) or a local host. A centralized system is much more limited in the way it interacts with the outside world because of its inability to devote large amounts of resources or to service any one special device.

- A distributed system is not necessarily subject to radical failure caused by the loss of a single machine. If the network is designed with adequate redundancy, the loss of any arbitrary number of machines and/or network links will not cause a total loss of functionality. The loss of the single machine in a centralized system is clearly catastrophic.

\section{Distributed Systems Architecture}

Much of the information available on distributed processing emphasizes the hardware and software technologies for data communications and computer configurations. There is significant emphasis now on providing sof tware for distributed data bases (DDB). Here we present and compare the major types of distributed systems with particular emphasis on designs that have potential application to nuclear materials accountability.

1. Interprocessor Communication. The task of transferring the data between the various elements of a distributed system is handled by a collection of hardware and software known as a network. Originally, networks consisted of computers communicating over phone lines via modems with little software protection or optimization. The simplest method is to use the standard serial communications lines that are used for terminal connections. Each device can be made to look like a terminal to the other device, and data transfer can be accomplished by sending American Standard Code for Information Interchange (ASCII) characters. This method works well for small amounts of data at low transmission rates. It requires that software on each end determine what is done with the data. The transmitted data may require manipulation to make them usable for the application program. More sophisticated networks perform many more housekeeping functions and present the data in a form readily usable by the applications.

Network advances complement the advances in microcomputers. Networks have evolved in both the hardware connecting the machines and the software protocols governing the network's behavior. Currently, many LANs are being connected by sophisticated media such as coaxial cables and fiber-optic links.

Some concerns in selecting, a network include the cost of transmission media and their installation, data-transfer rates, ease of interfacing, security, manufacturer support, ease of expansion and upgrading, and maintenance. Matching the application software to the network software can be a serious problem.

2. Interprocessor Cooperation. Multiple-processor computer systems may be characterized by the degree of interprocessor cooperation. There are three major levels of such cooperation: tightly coupled, loosely coupled, and uncoupled. Tightly coupled systems use common primary storage 
to solve common problems. Intermediate results are immediately available to the other processors to enable interleaved processing. Loosely coupled systems use communications media for sharing resources such as secondary storage, peripheral equipment, and interprocess communications. The separate processors work on programs that are related. In uncoupled systems, the computers work autonomously with no direct interconnection and transfer data between machines by magnetic tape, etc.

The distributed nuclear MC\&A system envisioned in this research would be of the loosely coupled type. The system would be a collection of autonomous machines collecting data from instruments, processing transactions, and performing other plant functions. Completed transactions would be transferred to the next higher level when requested by the higher-level machine.

3. Topology of Distributed Systems. The architectural feature that sets a distributed system apart from a centralized one is the medium used for communication among the computers in the distributed system. This medium is the network.

Distributed-processing systems can be categorized in a number of ways. Here we use the logical structure that is used to connect the machines. Martin 2,3 presents two broad divisions of network topologies: vertical and horizontal.

Vertical networks are characterized by having a hierarchy of machines in which transactions may enter the system at the lowest level, and the machine at this level can either process them or pass them up to a higher level. This logical arrangement separates the network into levels and gives it a vertical organization. An example of a vertical network is a star topology, as shown in Fig. 1.

Horizontal networks logically riew the machines as being at the same level. Any machine may talk to any" other without passing through some special intermediary. Examples of horizontal topologies are busses, rings, and meshes, as shown in Fig. 2. Often, groups of machines in a vertical network form a horizontal subnetwork. Therefore, some overlap between the two varieties is possible.

\section{Systems Design}

A properly designed distributed system must start with the objectives of nuclear materials accountability at the particular facility. The levels of resource and data sharing and processing must be carefully considered in how they can help attain the overall objectives. The system's architecture will depend heavily on how the objectives are to be attained, the characteristics of the data, and the information flow between application components.3,4 For example, a basic decision must be made on which applications are to be run on which network node. This decision depends on whether horizontal or vertical distribution is more applicable to a particular facility.

In addition, MC\&A systems have special requirements for the hardware and software because of their operating environments. The hardware must be able to withstand the industrial atmosphere of corrosives, moisture, heat, electromagnetic interference, vibrations, dirt, noise, etc. The software must provide security, reliability, near-real-time response, user friendliness, accuracy, and availability. 


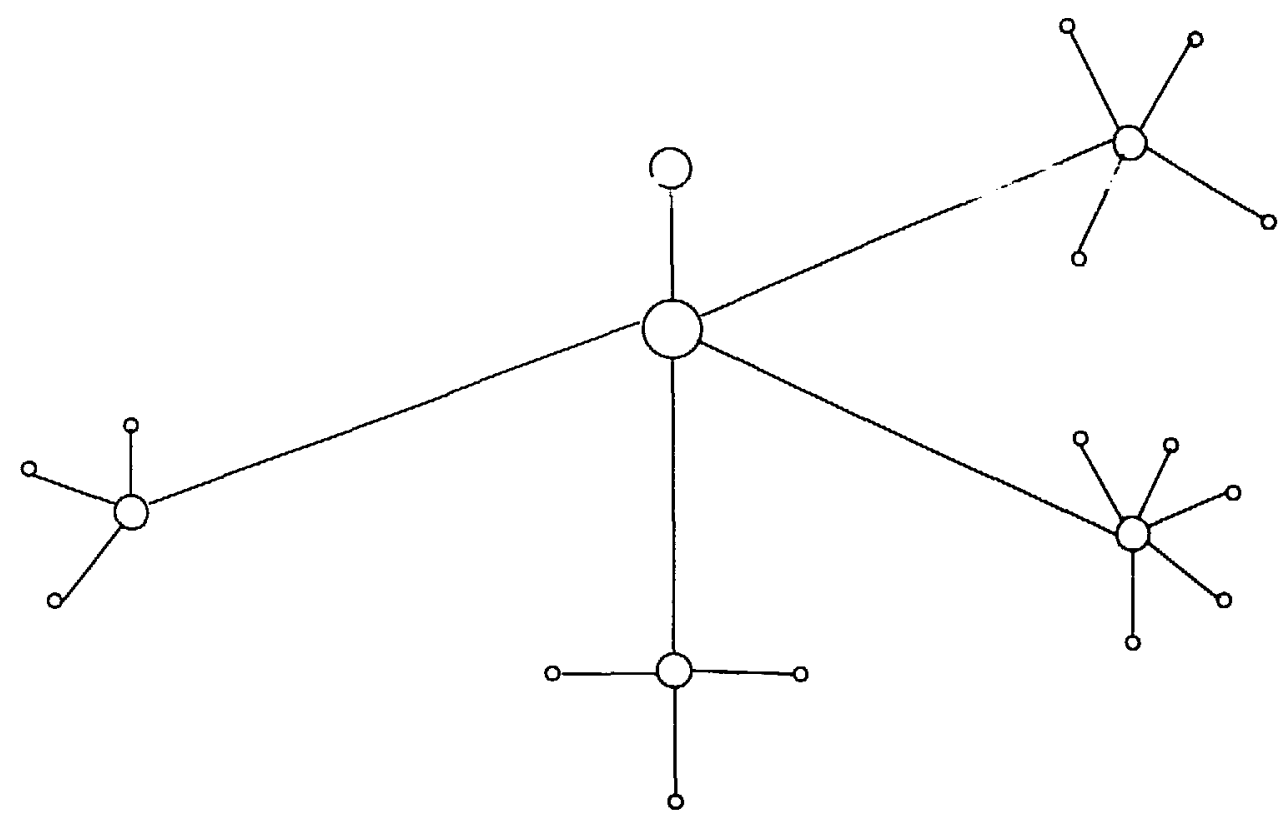

Fig. 1. A star as an example of a vertical network.
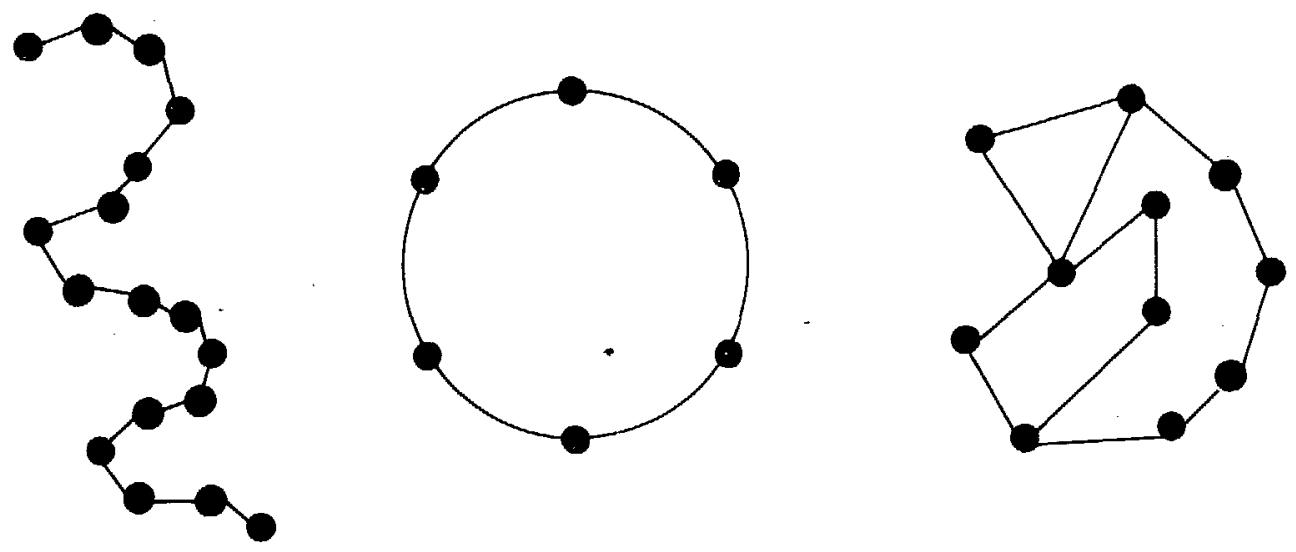

BUS

RING

MESH

Fig. 2. Examples of horizontal networks. 
Security and auditability controls are necessary with multiuser computer equipment, especially where DBMSs or other software products employ user-friendly modes of operation. Section II.F.3 discusses security.

Reliability is a primary concern. Shucting cown the accounting system because of isolated component failures is unacceptable. This shutdown will impact the design of the network, the number and type of computers, and the nature of the software's reliability features.

If near-real-time response is a goal, the use should be able to do real-time MC\&A functions without degrading system performance. This capability impacts on the data-acquisition systems, the data-base systems, the network, and the user's software interface.

User friendliness is necessary for system effectiveness. Operators should see the system as a boon to their jobs in the same way secretaries regard word processors. The system also should make the plant easier to run by removing much of the data-entry responsibility from the operators. This assistance will allow them to focus on work more directly related to nuclear materials processing.

Accuracy of the accounting data should improve because users will not be making measurement recording errors, reading labels incorrectly, etc. Also, computers can make many more measurements with equal or greater precision than can people. This capability will generate more and better data for the statistical packages and hence result in a better understanding of the overall material situation.

Availability of data for materials management should radically increase with a DBMS. The organization's management should be able to look at the appropriate amount of data for their needs because the entire plant's status may be obtained through the distributed DBMS.

\section{E. A Preliminary Configuration for a Distributed Nuclear MC\&A System}

From the previous sections, we have the ingredients for developing a preliminary design specification. Planning for a distributed nuclear MC\&A system starts with the statement of the objectives of the system. The general statement of overall objectives, 1 isted in Sec. II.A, suggests that a distributed approach is appropriate. The topology of a distributedprocessing system is related to the organization's hierarchical safeguards reporting structure. In a nuclear processing installation, daily information needs are unique for end-users, such as process control personnel and safeguards and security personnel. Next, we must consider long-term information requirements as well as current ones to propose an architecture for the distributed system. Finally, we must assess the technology for achieving the design. Here we focus on the informational requirements to suggest a reasonable distributed system architecture.

Process data are useful for production scheduling and reporting and long-term planning. In a we11-designed integrated safeguards system, much of the safeguards data may be obtained from the process data.

The above discussion suggests the use of the natural functional division of the information requirements between safeguards and processing. The safeguards information will pertain to the entire site. However, each different processing location will have a unique requirement. other process information, reiating to the overall processing complex, will be required for production planning and forecasting, preparation of reports, etc. Whenever possible, the physical security component of the safeguards system should be used. 
These informational requirements might exclude star structures, where all instruments are connected to the computer that also handles safeguards and production data processing. Mesh or network structures, rings, and shared line or bus structures might also be excluded because processors may be able to communicate with other processors when there is no need for communication. Having considered the limitations of these topologies at the overall system level, we find a hierarchical structure the most reasonable for a distributed nuclear MC\&A system because the data are distributed vertically. This vertical structure is based on the reporting structure derived from DOE requirements for central responsibility for a materials management program encompassing all organizations either handling or responsible for nuclear materials.5 In its simplest form, a hierarchical distributed system is shown in Fig. 3. Here, data at Level 1 are needed only at that local level. Transactions originating from terminals or other input devices connected to the computers at Level 1 are serviced at that level and uploaded to the computer at Level 2. Data at Level 2 can be downloaded to Level 1 for processing at that level.

A reasonable first design would be based on a modification of the simple system shown in Fig. 3. At the lowest leve1, microprocessors are used for data acquisition from NDA instruments, transaction processing and validation, and some plant production data processing.

In an environment with high transaction activity (>5000/month), multiple machines provide parallel input and processing of data. If the host machine fails, the low-level machines can continue to process transactions while buffering data for later update of the host machine's data base. Much of the activity of the low-level machines is routine housekeeping such as screen generation and data validation, which should not burden the host machine. The low-level machines serve for data input from the operators and/or on-line instrumentation. Screen menus and forms guide operators through the transaction processing. The intermediate-level machines serve as data buffers and repositories for data relating to single material balance areas (MBAs). They also provide computing power and resources for materials control, other integrated-safeguards functions, and potential benefits for process control. The high-level machine holds the official copy of the materials data base collected from all MBAs. This incormation is used for data analyses and generating official reports.

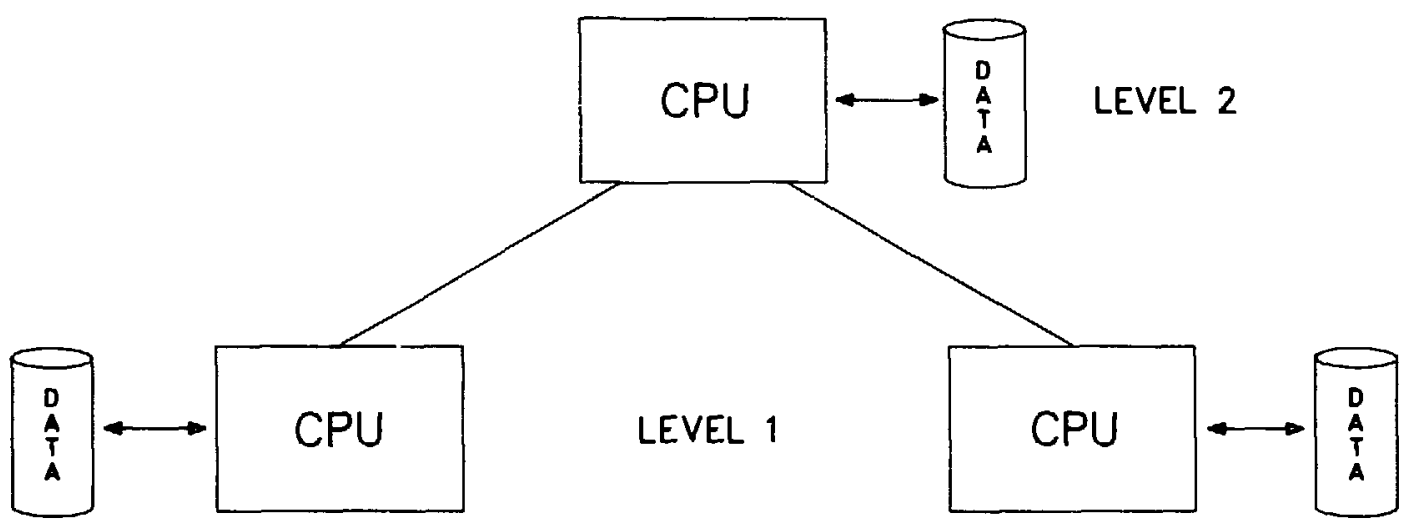

Fig. 3. Simple hierarchical distributed system. 
Figure 4 shows how a complete system might be structured for a large plant. As can be seen from tine figure, the logical structure of the system is a generalization of a star configuration. At the lowest leve1, microprocessors, possibly PCs, are used for data acquisition from instruments, transaction processing and validation, and some plant production data processing. Additional processing might be for line supervisors or staff personnel involved with plant production operations. Most data collected at Level 1 are passed to Leve1 2. Data stored in machines at Level 1 are necessary on $1 y$ at that level.

Leve1 2 is composed of larger microprocessors and/or minicomputers. A reasonable projection for the next few years is that technical workstations could serve as Level 2 machines. The workstations could contain the main peripherals for sharing with the machines at Level 1 . Data validation and data-base update, which require much more processing capacity than available on the PC-1ike devices at Leve1 1, could either be handled at Level 2 or at Level 3. Finally, pertinent data are uploaded to the main machine at Level 3, which probably is a machine of a higher class than at Level 2.

The main data base resides at Level 3. Regular safeguards and security operational processing such as preparing reports to DOE occurs at this level. Requests by Level 2 machines for data stored in the main data base are downloaded to the requesting machine. These data, in turn, could be downloaded to a machine at Level 1 . Machines at Levels 1 and 2 use DBMSs for ease of data handling, analyses, and reporting. Proper data-base design and the selection of a DBMS package greatly influence the ability to produce special analyses and reports either through ad hoc queries and default reporting procedures or through menu- and forms-driven interfaces.

The main machine may use special-purpose software for production runs and a DBMS-1ike product for information analyses. Possibly more than one machine could be needed at Level 3, one for production-applications software and the second machine with another copy of the data base accessible by a DBMS for special analyses/reporting and ad hoc queries.

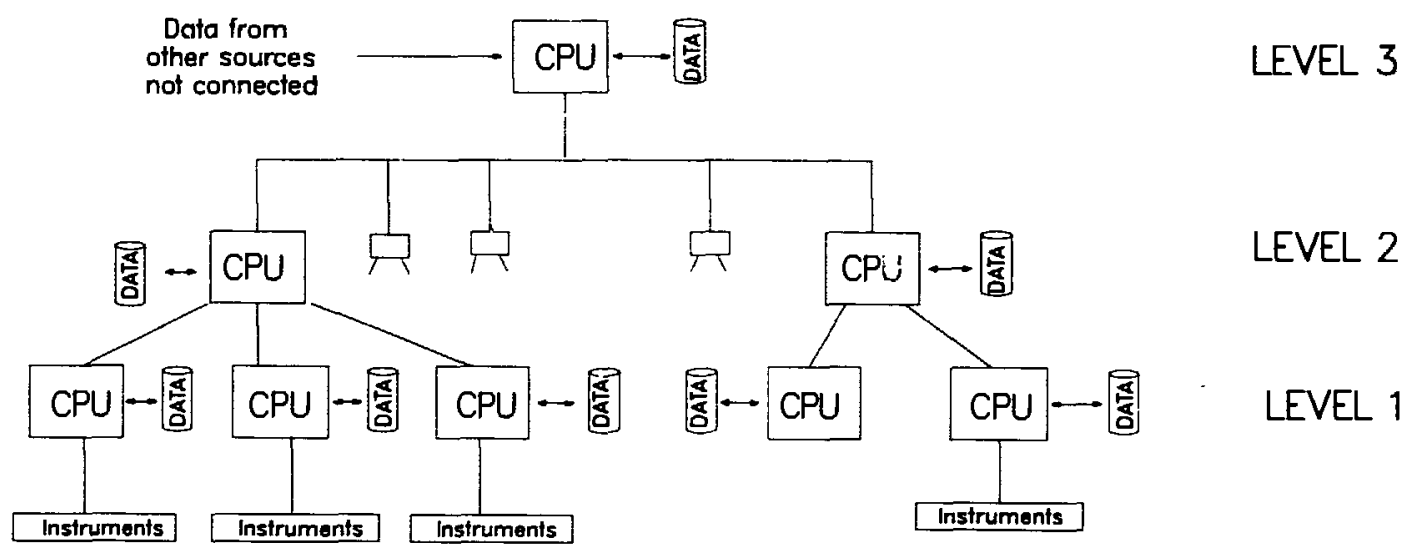

Fig. 4. A generalized distributed MC\&A system. 
The host machine is a genera1-purpose computer with the computational speed to provide adequate throughput with the required response using the selected software. Capacity must be adequate for present needs plus projected increase over the expected system life. However, most expansion should occur at lower levels in the architecture. Very small facilities with minimal requirements may be adequately serviced by a PC. Larger facilities with high throughputs may require a supermini or even a mainframe for the main computer, depending on traffic and reporting requirements.

\section{F. Design Considerations for the Preliminary Configuration}

The preliminary design as shown in Fig. 4 and related narrative describe a logical structure and data-storage possibilities for a distributed nuclear MC\&A system. This section describes the considerations that must be given to data acquisition, I/O devices, data storage, computer security, data-base protection, routine and special reporting, system performance, and software selection in designing a distributed nuclear MC\&A system.

1. I/0 Device Use. There are many special considerations for selecting $\mathrm{I} / 0$ devices in nuclear materials accounting. These devices can include the following: (1) PCs for data acquisition, (2) PCs for supervisor use, (3) smart terminals for data entry and ad hoc reporting, and (4) video display terminals for presenting process or safeguards information. Not only do the machines need to be durable and reliable, but they must also be matched to specific tasks to ensure that these tasks can be performed satisfactorily without causing major security problems. Some of the local instruments may be connected to PCs.

2. Disk Storage. Disk storage should not be allowed where there is no need for it. For example, PCs used exclusively for data acquisition/ communication should provide limited access to the data to the extent necessary for the device(s) to function properly. The supervisor, however, may need some storage, but other data, where there is not day-to-day need, should be stored at a higher level. In other cases, a PC may belong to a process supervisor. Here, more of the data base will need to be accessible. The easier the data-base language is to use, the more restrictions there must be on access to I/O devices that have access to the storage medium.

3. Computer Security. Security is a very difficult and complex issue when dealing with modern computer systems. Computer security is widely discussed in the 1iterature. Therefore, we will mainly explore the additional security aspects imposed by a distributed system.

Security for computer systems is primarily aimed at protecting the data from unauthorized disclosure, modification, or destruction. The same type of security is necessary for a distributed system, but the means of providing this security may be more difficult because distributed systems increase the potential accessibility to the data.

Gaining unauthorized physical access to a computer terminal or other I/O device is a physical security problem. Security controls provided by the computer system involve access control and authorization control. 
Gaining access to the computer system involves some means of user identification. If an intruder is identified as a valid user, he can do anything that that valid user is allowed to do.

Movement of data sets across a network poses more security concerns than the possibility of individuals gaining access to individual records. With older data-transfer and computer systems, individuals had to acquire printouts, magnetic tape, or disks or had to commit a security violation to get unauthorized information. Electronically tapping the network was not much help because only individual records could be obtained.

The widespread use of intelligent terminals and microprocessors can allow access to the data base by applications programs, query languages, report generators, and DBMS utilities. Relational DBMSs pose some security problems because they employ nonprocedural languages, which allow a user to access data without knowing the complex logical or physical structure among the data items. One of the most common weaknesses of many DBMSs to penetration is that they run under control of operating systems. Present operating systems and DBMS packages are just beginning to incorporate more stringent security features.

There is disagreement on whether distributed systems are less secure than centralized ones. Protection of data in computer systems that do not use data-base techniques is more difficult in some ways; many files with redundant data must be protected. Conversely, many files may require accessing to produce the sought-after information--a distinct disadvantage for the intruder. However, DBMSs that have well-designed security features can be more secure than file-management systems.

It is not known which distributed data-base topology offers the best security. However, a distributed system with a centralized data base using a flexible, user-friendly DBMS allows access to all the data if the hardware/software protection can be violated. Without tight controls designed into the hardware and software, other security precautions will not ensure the level of security needed.3,6,7 It may be possible to make a distributed system more secure by partitioning the data among different machines. Data can be partitioned either locally or global1y. Local data are used on one computer of the distributed system. Global. data are used on two or more machines. Judicious partitioning of the data can go a long way in aiding security.

Because the data-processing environmeni has a great influence on software requirements, it is difficult to list the security features of a distributed nuclear MC\&A system. These features will vary with the degree of data sharing and the sensitivity of the data.8 However, the security must begin in the operating system software and the DBMS(s). The concept of data sharing among users and applications in a system of any size poses severe security problems because the security system designer must be familiar with all of the features of the operating system as well as the features of the DBMS(s).

A secure system needs layers of protection. The two outermost layers are physical security and administrative security. $3,6,8$ Audit controls can be resource consuming if carried too far. Audit trails could focus, principally, on randomly selected data items or on items where evidence suggests a specific area where further investigations might prove fruitful. If necessary, encryption can provide more security for sensitive data 
transmitted over communication lines that allow data to be accessed outside the DBMS. For a distributed nuclear MC\&A system, security issues must be carefully examined.

4. Data-Base Protection. The loss and/or corruption of data in a data base are two of the major problems that must be addressed. Loss and/or corruption can be intentional as well as unintentional. In addition, steps must be taken to prevent erroneous data from being entered. After security procedures have been applied, allowing the user to have access to a portior of the data base, the first step in preventing erroneous data from entering the data base is local error checking. Local error checking can include data validation by field size, field type, range of values, and additional software routines that may use artificial intelligence techniques to ensure transactions are valid.

All of the above issues are problems of data integrity, which refers to the reliability of the data as they are stored in the data base. Proper data-base design plus the judicious selection of DBMS software will alleviate or reduce many data-integrity problems.

Preventing corruption of the data base is influenced by whether the data are shared or are private. Some of the data at Level 1 may be private. In most cases, however, the data will be shared. Creating or using an available data dictionary utility can aid in keeping track of where data are used and identifying or assigning them shared or private access. Another consideration, although not related to corruption or loss, is the currency of the data. Currency requirements must be carefully examined in the systems design. This examination includes a cross check of data currency needs with shared usage. A fundamental principle that should be adhered to is that once a data element is placed in the data base, it should never have to be reentered. In addition, it should not be possible to alter entered data, especially data stored at Level 3, except through a valid trarsaction.

Accidental loss or corruption of data caused by hardware or sof tware failures can be protected against by using a DBMS package with good restart and recovery capabilities. However, planning for the proper use of these utilities is an important consideration.

Proper data-base design is crucial for security, data integrity, and performance. Improper design that does not take into consideration data independence can lead to redundant data with the consequence of potential inconsistency of data. Insertion and deletion of data are also problems with an improperly designed data base.

A discussion of relational data-base design is beyond the scope of this report. However, protecting the data base against misuse comes down to two issues: (1) integrity preservation and (2) security or access contro1.

5. Routine Reporting. Routine reporting occurs at all levels. At the lower levels, reporting is directly related to production, inventory, and forecasting. Much of this information is private--belonging to one or a few production supervisors. 0ther data are downloaded from the highestleve1 machine to produce information at lower levels. Some of these could be inventory data to produce, for example, a report on scrap material stored in a vault. This type of report would help production supervisors schedule scrap material in production runs. 
A thorough analysis of the informational requirements must be conducted. The technique used for analyzing user requirements and designing a system to satisfy these requirements is called structured design. Structured design aids in producing correct systems by partitioning the overall problem into simpler components and relating these components in a hierarchical organization.

6. Special Reporting. Proper data-base design and the selection of a DBMS package greatly influence the ability to produce special analyses and reports either through ad hoc queries and default reporting procedures or through menu- and forms-driven interfaces. Flexibility and productivity are the hallmarks of modern relational DBMSs.

Special reporting requirements at the two lower levels can best be served by any number of available DBMSs. For the Level 3 machine, special reporting procedures are complicated by the type of software used for normal data processing. If special-purpose software is used for production using data in a file-structured system, the ability to generate ad hoc queries, reports, and analyses will depend on the availability of other software supplied by either the computer manufacturer or another software vendor.

7. System Performance. Nuclear MC\&A systems at different facilities will have different characteristics primarily related to the mission and size of the facility. - Fundamental characteristics of the s-feguards and security component, however, will be similar in that DOE Orders and reporting requirements must be met. Two basic factors affect the capability of the MC\&A system to perform its function in a timaly manner--transaction volume and system design. Transaction volume is a characteristic of the site, and the system design must carefully provide for hand?ing the current transactions and provide for expansion. The trinsactien volume, the need for timely information, and providing for expansicn have a direct bearing on the types of hardware and software used to implement the design. The selection of these hardware and software types also depends on whether the highest-ievel system uses special applications software or a generalpurpose DBMS and on the partitioning of the data. (Partitioning refers to the assignment of data to processors and establishing boundaries in the sof tware--which does what and when.)

In large facilities with very large data bases, data-base machines may provide the required performance for production systems. A data-base machine is a specialized combination of hardware and relational DBMS software that can improve the performance of the system over available relational DBMS software, relieve other equipment from CPU and storage overuse, and tie together heterogeneous computers.

There are several disadvantages of data-base machines. Their future is unclear and they are expensive. However, the advances in data-base machine technology should be examined for evaluating its use in MC\&A systems.

8. Special-Purpose Software vs Commercial DBMS6. With the commercial realization of the DBMS concept in the early 1970s, a fundamentally new approach to data processing occurred, which was data-centered rather than program- or application-centered. Since the mid-1970s, there has been an increased interest in relational DBMS, and now virtually every vendor 
is claiming relational or relational-like systems. Relational data bases offer many advantages over cther data models. Some of these advantages include simplicity, ease of extension, data independence, and ad hoc query capability. However, relational DBMSs are of ten slower than DBMSs based on other data models, although great strides are being made to improve the performance of relational DBMSs. Some applications cannot use a generalpurpose DBMS but require special-purpose software to meet their needs.

A major problem in distributed processing is the incompatibility of data on different hardware and operating systems. Also, network architectures used to interconnect machines are not always compatible. In some cases, software may need to be written to allow the connection of machines. In other cases, software may already be available; large DBMS suppliers are marketing interfaces between their software products and the PC and other computer software products. In addition, RTI and ORACLE claim to have distributed software as well as their DBMSs running on a wide variety of machines.

In addition to the complications of interconnecting machines and the ability of software on different machines to transfer data in a useful form, a primary concern is the ability of a general-purpose, commercial DBMS to perform the main processing tasks adequately. Commercial DBMSs must be general enough and have enough features to satisfy a wide market if they are to succeed commercially. This requirement means they have many features not needed for a particular site. These excess features take up space and have the potential of degrading response time and increasing the difficulty of use. A commercial DBMS may not be satisfactory for production-type processing hut may be the preferred method for special analyses, error checking, ad hoc queries, and bulk transaction handling. In the latter case, transactions would be processed at a local node, and then a number of them would be uploaded to the main computer. In some cases, a commercial DBMS could be used to transfer the data.

Processing on the main computer likely will include special analyses and ad hoc queries as well as production-type processing. In this case it could be necessary to provide two copies of the data. One copy would be used for production with special-purpose software, and the other copy would use a commercial package for special information handling. This method requires redundant data with, in addition to the additional cost of storage, the possibility of some data not being current.

Many data-processing professionals are now changing from the single data-base to the dual data-base concept. One data base would be used for on-line or operational applications, and the other data base would be used for decision support or information retrieval. Operational applications is usually very structured, whereas the decision-support type of operations is usually very unstructured. With dual data bases, separate machines would be required to get the most out of the processors. However, with the dual data-base concept comes the problem of keeping the data current and correct in both data bases. A single-record entry system in the operational system is the appropriate method to overcome these problems. The decision-support data base would derive its data from the operational system. The operational system would be on a mainframe or superminicomputer, whereas the decision-support system would be on a minicomputer or a microcomputer. 


\section{SYSTEM DEVELOPHINT}

\section{A. Hardware Selection}

The selection of hardware for an MC\&A system should be determined by a host of considerations including

(1) selection of software,

(2) functionality,

(3) maintainability,

(4) availability,

(5) ease of use,

(6) cost effectiveness, and

(7) compatibility with environment.

These are the normal considerations used in the selection of any computer system but must be interpreted in light of stringent local plant requirements.

Selection of software is a strong factor, though not complecely dominant. The software industry is so competitive today that there may be a number of packages that meet the requirements. This abundance allows more flexibility in selecting hardware. Availability, ease of use, and cost effectiveness are the normal economic and ergonomic constraints. Functionality would indicate the selection of an open-system style of architecture to accommodate equipment from a wide variety of vendors. If in-1ine equipment is to be installed from a number of different vendors, this equipment must be capable of communicating with the controlling processor. Open systems also foster the development of third-party boards and equipment that greatly simplify the process of system development.

Maintainability means that the system can be returned quickly to a ful1-functioning state after a malfunction. This can be achieved by having rapid response from the servicing organization, built-in redundancy, or quickly repiaced spares. In the latter two cases, the service still must be reasonably fast. A distributed network with adequate buffering of data can relieve somewhat the requirement for rapid response. Failure of one component will degrade the functioning of the system but will not cause complete system failure.

Special-purpose hardware is justified only in cases where no commercial. ccmponents are available for a specific purpose. This may be the case with special in-1ine assay or measurement instruments. The market provides considerable selection in general-purpose computers and networking equipment at reasonable prices. Custom development of such equipment is not cost effective.

There are four levels to consider in configuring the hardware of a distributed system:

(1) instrumentation,

(2) terminals,

(3) communications, and

(4) host machine.

The instrumentation shown in Fig. 4 includes devices such as balances, bar-code readers, fluid-level indicators, counters, assay equipment, pressure transducers, and voice $\mathrm{I} / 0$ equipment. Most commercial instruments of these types are now available with Electronic Industries Association RS-232-C or IEEE-488 computer interfaces. Custom assay instruments usua11y incorporate microprocessors that inherently include computer interfaces. 
Terminals range from simple keyboards with monitors, through intelligent terminals, to complete microcomputer.

Communications requirements are determined primarily by software design and throughput. Several de facto standards have recently emerged with differing levels of performance. New hardware and software packages are beginning to agpear for these new standards and will provide considerable selecticn. As networks become more widespread, costs will continue to decline. Additional information on communications is provided in the following section on networks.

The host machine is a general-purpose computer that complies with the initial seven considerations enumerated above. Capacity must be adequate for present needs plus projected increase over the expected system life. If nonstop computing is a firm requirement, the selection is limited to a few vendors. Small facilities with minimal requirements may be adequately serviced by a PC-class machine, whereas the largest facilities with high throughput may require a supermini or a mainframe.

\section{B. Networks}

A large number of manufacturers have designed and promoted networks. Usually, these were specialized to run on a limited set of hardware and interact with limited software. Because of the incompatibility problems, the International organization for standardization set out to define a standard for data communications. The result was the issuance of the Open Systems Interconnection (OSI) reference mode1.9,10 This model organized the task-to-task communications problem into seven layers, encompassing all the hardware and software required. Each layer is designed so that changes can be made with minimal effect on other layers. Separate standards can be established for each layer. The lowest level is the physical connection, and the highest level is the connection to the application program. So far, only the two lower levels have reached the final stages of standadization.

An interconnection method should be selected that conforms to the OSI model to obtain the greatest flexibility, growth potential, and likelihood of widespread support. More and more manufacturers are recognizing the advantages of conforming to such standards. Selecting a network supported by a single manufacturer may result in compatibility problems, limited flexibility, and early obsolescence. The OSI model does not preclude any physical medium or topology. These can be selected to best meet the requirements of the installation. Upgrades or expansions can be made without disturbing the entire network system.

Many products are on the market that are called LANs or that include network or net in the name and description. Usually these products only incorporate the hardware portion of the OSI model (physical and data-1ink layers), although some incorporate the next two layers in software. Care should be taken to determine what the product actually includes. Selecting one that meets one of the options under IEEE Standard 802.X will ensure widespread support. Three principal choices for the physical medium, listed roughly in order of increasing cost and bandwidth, are twisted pair wires, coaxial cable, and fiber-optic cable. Media cost and installation may be a small fraction of the network costs, and false economy may result in early obsolescence. 


\section{Data-Processing Software}

In this context, data-processing sof tware means any sof tware running on any machine supporting data processing. It specifically excludes any commercial or special-applications software that supports networking as its main function. It does not exclude DDB or network support components of, for example, relational DBMS packages. Special-purpose applications sof tware may need to be developed for production runs on the main computer. Other special-purpose sof tware also may be needed at other nodes.

Connectability is one of the key issues for organizations using heterogeneous computer systems. Not all vendors can connect their own systems; IBM is an example. Because of the potential of different machines being used in a distributed nuclear MC\&A system, it is necessary to evaluate DBMSs that can communicate with data bases on machines of different manufacture.

Some relational DBMSs may place an undue burden on a system designed for production when used for ad hoc analysis and reporting requirements. Limited production-type reporting probably would not suffer much, especially if run on off-load hours such as at night or on weekends. Performance enhancements occurring in the next few years probably will allow relational DBMSs to perform very similarly to CODASYL, network, or hierarchical DBMSs in production environments. Still, the possibility of special-applications software must be considered for production, especially if the main computer is not a mainf rame.

\section{INTEGRATED SAFEGUARDS}

Distributed-processing technology has the potential of enhancing not only MC\&A systems but also the entire area of integrated safeguards. With proper attention to safeguards and data-processing systems design, dịferent organizations having different safeguards and management responsibilities could coordinate the data and information collected to provide a much more highly integrated capability than now exists. A single data base, distributed by organizational function, could include materials accounting, materials control, and authorization of individuals to access data, material, and locations. These data could provide decision makers with timely information that would allow more rapid response than is available now. Distribution of data, with proper systems design, would allow each separate organization to control its data and secure its own functions as authorized by top management.

\section{RECOMMFNDATIONS FOR SAFEGUARDS RED}

The development of efficient and cost-effective safeguards information systems using networking and distributed-processing technology should rely on design concepts, methodologies, and design tools developed by the general computer science and applicativis programming profession. In this section we address areas that are specific or unique to the safeguards applications and best developed by the safeguards community in cooperation with computer science professionals. Work undertaken by the safeguards community should provide technical support for 
- DOE headquarters' needs in establishing policy and long-range planning,

- field-office evaluation of existing materials accounting systems and proposals of major construction or upgrade projects, and

- facility-specific problems or initiatives in the area of safeguards information systems.

A major consideration in designing any transaction-based system is to match the hardware and software to specified performance requirements. A basic set of design criteria for implementing a distributed MC\&A system should be developed, both as a separate materials accounting system and as an integrated safeguards system. The concept design provides a framework for further system study and for the development of specific applications that require tailoring to meet site-specific functional requirements. System design tools, such as cost models used to match various hardware and software configurations to performance requirements tailored to the safeguards information systems, would provide useful tools in developing efficient and cost-effective systems.

System design takes as input a description of the informationprocessing and security requirements of a particular MC\&A application together with the performance characteristics of candidate hardware and software configurations. Consequently, an ongoing effort to evaluate commercially available DBMSs, networking hardware and software, and workstation performance characteristics with respect to specific design requirements should be maintained.

Security is a special concern where networks and user-friendly modes of operation are used. A distributed system provides increased potential accessibility to the data and requires more care to protect the data from unauthorized disclosure, modification, or destruction. The possibility of insider threat poses additional concerns about the security of the safeguards systems and becomes increasingly important when safeguards activities are integrated througn a single information system. Relationa1 DBMSs pose security problems because they employ nonprocedural languages, which allow a user to access data without knowing the complex logical or physical structure among data items. The widespread use of intelligent terminals and microprocessors provides access to the data base by applications programs, query languages, report generators, and DBMS utilities. The security measures required to meet DOE regulations concerning the protection of classified information and the implication of information security agains $\hat{t}$ the threat of nuclear materials diversion or theft needs to be evaluated.

The construction of a test-bed for distributed-processing research in MC\&A, including appropriate hardware and software, has been shown to be useful for providing direction for the design and acquisition of advanced MC\&A systems. Increasing the size of the test-bed, testing and adding new commercial software including distributed DBMSs, evaluating other networking products, and testing different distributed architectures are areas that should be investigated. 


\section{vI. CONCLUSIONS}

Recent advances in data-processing and conmunications hardware and software, coupled with more favorable pricing policies, make distributedprocessing concepts attractive research prospects for advanced nuclear MC\&A systems. We believe that a properly designed MC\&A system based on distributed processing could increase the performance of transaction-based operational data processing and provide the capability for special analyses and ad hoc queries. The primary reasons for using distributed processing are

(1) expandability,

(2) improved system response, and

(3) decreased vulnerability to machine malfunction.

Microprocessors are useful at lower levels in a distributed system for data collection, local transaction handling, process control support, and as terminals for computers at higher levels. Other advantages include resource sharing (disks and data) and reducing the load on the main computer in terms of $\mathrm{CPU}$ use and storage requirements.

One major problem in designing distributed systems is being able to connect different computers using different operating systems. However, many software suppliers are concentrating on solving the connectability issue by providing software packages that allow connections between different machines and on DDB software. Additional effort is taking place on the network leve1.

There are enough commercially available, attractively priced products, both hardware and software, that there is no justification for large-scale development of special-purpose data-acquisition, data-management, networking, and related software products. Certainly, some special-purpose NDA instruments and come special-applications software may be necessary in certain instances.

The construction of a test-bed for distributed-processing research i:l MC\&A, including appropriate hardware and software, has been shown to be useful for providing direction for the design and acquisition of advanced MC\&A systems.

Security is a special concern where networks and user-friendly modes of operation are used.

The culmination of this work should be a set of design criteria for implementing a distributed MC\&A system either alone or as a part of an integrated safeguards system. The application of the concepts described here would require tailoring to meet site-specific functional requirements.

\section{ACKNOWLEDGIENTS}

We thank L. Y. Williams for preparing the graphics, S. L. Hurdle for compiling the manuscript and graphics, and B. W. Burnham and R. E. Lewis for their suggestions. 


\section{REFERENCES}

1. D. G. Shirk, R. C. Bearse, R. S. Marshall, A. I. Baker, and C. C. Thomas, Jr., "Los Alamos Plutonium Facility Applied Systems Integration Project Status Report for Period Ending August 31, 1981, "Los Alamos National Laboratory report IA-9224-SR (February 1982).

2. J. Martin, Computer Networks and Distributed Processing (Savant Research Studies, Carnforth, Lancashire, England, 1979), pp. 29-37.

3. J. Martin, Design and Strategy for Distributed Data Processing (Prentice-Hal1, Inc., Englewood Cliffs, New Jersey, 1981), pp. 120$162,347-374$.

4. J. T. Siarkin, C. A. Coulter, R. G. Gutmacher, C. C. Thomas, Jr., and W. J. Whitty, "Design and Evaluation of an Integrated Safeguards System, Volume I: Principles," Los Alamos National Laboratory report LA-9964, Vo1. I (March 1984).

5. Orders 5630.1 through 5630.8, US Department of Energy, Office of Safeguards and Security, Washington, DC.

6. J. Martin, Corporate Strategy for Distributed Processing (Savant Research Studies, Carnforth, Lancashire, England, 1979), F, 169.

7. J. Martin, Distributed Processing - Network Mechanisms, Standards and Recovery (Savant Research Studies, Carnforth, Lancashire, England, 1979), PP. 307-310.

8. J. King: Evaluating Daca Base Management Systems (Van Nostrand Reinhold Company, New York, 1981), Pp. 182, 252.

9. International Organization for Standardization, "Information Processing Systems - Open Systems Interconnection - Basic Reference Mode1," International Standard 7498, Geneva, Switzerland (1984).

10. J. D. Day and H. Zimmerman, "The OSI Reference Model," Proc. IEEE 12 1334-1340 (1983). 